\title{
Un enfoque de ingeniería de requerimientos basada en el alineamiento de almacenes de datos y la estrategia del negocio
}

\author{
A requirements engineering approach based on the alignment \\ of data warehouses and business strategy
}

\author{
Ania L. Cravero $^{1} \quad$ Samuel E. Sepúlveda $^{1} \quad$ José N. Mazón $^{2} \quad$ Juan C. Trujillo² \\ Recibido 10 de mayo de 2012, aceptado 29 de abril de 2013 \\ Received: May 10, $2012 \quad$ Accepted: April 29, 2013
}

\begin{abstract}
RESUMEN
Garantizar que los almacenes de datos estén alineados a la estrategia del negocio es primordial para su éxito, ya que estos son utilizados por los gerentes del negocio con el fin de analizar los datos estratégicos de la organización. En este trabajo presentamos un enfoque de ingeniería de requerimientos orientado al negocio que alinea el Almacén de Datos a su plan estratégico. El proceso se describe mediante un conjunto de directrices que incluyen: el análisis VMOST para obtener los objetivos desde los usuarios, el modelo BMM para comprobar que los objetivos definidos estén alineados con la estrategia, el modelado de objetivos por medio de $i^{*}$ con el fin de obtener los requerimientos de información del Almacén de Datos, y el modelado multidimensional mediante un perfil UML. Se presenta un estudio de caso para mostrar el proceso completo.
\end{abstract}

Palabras clave: Almacén de datos, alineamiento, directrices, estrategia del negocio, ingeniería de requerimientos.

\begin{abstract}
Ensuring that data warehouses are aligned to the business strategy is critical for their success, as these are used by business managers to analyze the organization's strategic data. An approach to requirements engineering-oriented business that aligns the data store to its strategic plan is presented. The process is described by a set of guidelines that include: VMOST analysis to obtain the objectives from the users, the BMM model to verify that the defined objectives are aligned with the strategy, modeling objectives through $i *$ to obtain the information requirements of the data warehouse and multidimensional modeling by UML profile. A case study to show the entire process is presented.
\end{abstract}

Keywords: Data warehouse, alignment, guidelines, business strategy, requirements engineering.

\section{INTRODUCCIÓN}

Los Almacenes de Datos (AD) son utilizados por los sistemas de toma de decisiones con el fin de analizar un gran número de datos provenientes de diversas fuentes heterogéneas, integradas en un modelo multidimensional (MD) [1-2]. Los diseñadores en general muestran los datos en los hechos y las dimensiones desde un MD conceptual. Habitualmente la información almacenada en los hechos representa medidas para los procesos de negocio (por ejemplo, ¿cuántos productos se venden? ¿Cuántos pacientes son tratados? ¿Cuánto tiempo se requiere en un proceso determinado?, etc.) y las

\footnotetext{
1 Centro de Estudios en Ingeniería de Software (CEIS). Departamento de Ingeniería de Sistemas. Universidad de La Frontera. Av. Francisco Salazar 01145. Temuco, Chile. E-mail: ania.cravero@ufrontera.cl; samuel.sepulveda@ceisufro.cl

2 Grupo de investigación Lucentia. Departamento de Lenguajes y Sistemas Informáticos. Universidad de Alicante. Apto. de correos 99. E-03080. Alicante, España.E-mail: jnmazon@dlsi.ua.es; jtrujillo@dlsi.ua.es
} 
dimensiones representan el marco para el análisis de estas medidas (por ejemplo, tiempo, cliente o producto) $[1,3-4]$.

Tradicionalmente el desarrollo de los MD conceptuales han sido guiados por un análisis detallado de las diversas fuentes de datos. Sin embargo, varios estudios han señalado que la mayoría de estos MD no abordan la información estratégica requerida por la organización como consecuencia de una mala comunicación entre los diseñadores del AD y los tomadores de decisiones [5-9]. Esto debido a que la definición de los objetivos a alcanzar, y los requisitos de información del AD son obtenidos de forma manual dependiendo de la destreza del diseñador $[5,10,11]$.

En este contexto, el objetivo primordial de la ingeniería de requerimientos para un $\mathrm{AD}$ es representar a los usuarios, sus objetivos y las relaciones entre los mismos, con el fin de alcanzar los objetivos estratégicos del negocio [2, 12-16]. Esta etapa es crucial en el desarrollo del AD, ya que generalmente las partes interesadas presentan necesidades que están relacionadas con sus propios objetivos y no a los organizacionales y, por tanto, el AD final puede no estar alineado a la estrategia del negocio [9, 17-20]. Por lo tanto, esta etapa permite a los desarrolladores ubicar el AD en el contexto de la organización y alinearlo con sus objetivos estratégicos [21].

Por otro lado, la revisión de la literatura demuestra que muchos desarrolladores no ponen ningún énfasis en la necesidad de alinear su AD con los objetivos del negocio, lo que podría sugerir que los equipos de proyectos no se animan a pensar en el AD como una herramienta estratégica $[9,17,22]$. La fuerte reducción en el interés de que los proyectos de $\mathrm{AD}$ deben alinearse con la estrategia organizacional y los objetivos del negocio es preocupante $[9,22]$. Weir, Peng y Kerridge [9] concluyeron que un AD debe tener un propósito claro para el negocio y que la organización debe ver la tecnología como un motor para la mejora en la toma de decisiones y el desarrollo de sus negocios.

Cooper [21] demuestra que es posible lograr el alineamiento entre el AD y los objetivos estratégicos del negocio por medio de algunos casos de estudio.
Por otro lado, Simonin [17] utiliza un método para diseñar AD alineados a la estrategia del negocio mediante un conjunto de reglas y patrones. Otro trabajo es el de Kumar [13], quien desarrolla un conjunto de criterios de calidad para diseñar un AD considerando los objetivos de decisión del negocio como base, pero no es un método de alineamiento. En Shanks [23] identificaron una serie de factores críticos de éxito para la implementación de sistemas de análisis del negocio en general, por medio de un ejemplo con AD.

En este sentido, el grupo de investigación Lucentia [2, $15,16,19-20,24-25]$ se ha preocupado por diseñar $\mathrm{AD}$ en el contexto organizacional, por lo que lleva tiempo desarrollando y mejorando una propuesta de ingeniería de requerimientos para $\mathrm{AD}$. En este trabajo presentamos un enfoque de ingeniería de requerimientos orientado al negocio, definiendo un conjunto de directrices para diseñar el AD alineado a la estrategia del negocio. Las directrices incluyen (i) el análisis VMOST [26] para obtener los elementos de la estrategia del negocio desde los usuarios del AD (objetivos), (ii) el modelo BMM [27] para comprobar que los objetivos definidos estén alineados con la estrategia de la organización, (iii) el modelado de objetivos por medio de $i^{*}$ [28] con el fin de representar los objetivos y relaciones entre los distintos usuarios, y (iv) el MD del AD mediante un perfil UML [15].

El trabajo se encuentra estructurado de la siguiente forma: primero damos a conocer los conceptos básicos de un AD, por otro lado, en la sección siguiente se describen los trabajos relacionados. A continuación, se presenta nuestra propuesta de ingeniería de requerimientos para AD. Por otro lado, presentamos las reglas de VMOST y BMM, además de un conjunto de directrices para alinear el AD a la estrategia organizacional. Luego, se describe un estudio de caso. Finalmente, se explica las principales conclusiones obtenidas.

\section{CONCEPTOS BÁSICOS}

La definición clásica de AD fue acuñada por Inmon [29] como una colección de datos históricos, orientados por temas, no volátiles, integrados, diseñados para apoyar el proceso de toma de decisiones de una organización. 
Más que una simple recopilación de datos, el AD surge de un proceso definido en tres etapas: (1) extracción de datos de distintas fuentes de datos, (2) la transformación y carga de datos de forma coherente en el AD [1] y (3) el acceso a los datos integrados de una manera eficiente y flexible. Las dos primeras etapas forman parte del proceso conocido como Extraction-Transformation-Load (ETL).

La principal contribución de un $\mathrm{AD}$ es su capacidad de convertir datos en información estratégica, apoyando la toma de decisiones en los niveles más altos de una organización. Esta capacidad se apoya en la herramienta OLAP [29], que proporciona a los usuarios finales vistas configurables de datos desde diferentes ángulos y en diferentes niveles de agregación.

Para lograr consultas OLAP de manera rápida y flexible, los datos están organizados en una forma multidimensional, conocido como esquema estrella, donde la información se clasifica de acuerdo con los hechos y dimensiones [29]. Los hechos son los datos numéricos o de hecho que representa una actividad industrial específica que se desea analizar. Las dimensiones son las perspectivas individuales de los datos que determina la granularidad (datos a nivel de detalle) que se adopten para la representación de un hecho. Las unidades de los hechos y sus valores se denominan medidas [29]. La Figura 1 muestra el proceso completo.

\section{TRABAJOS RELACIONADOS}

Es importante destacar que la etapa de ingeniería de requerimientos para un $\mathrm{AD}$ debe basarse en un marco orientado a objetivos (GORE) [30], ya que (i) el AD tiene por objetivo proporcionar información adecuada para apoyar la toma de decisiones, contribuyendo así a cumplir los objetivos estratégicos de una organización, (ii) los requerimientos del $\mathrm{AD}$ son difíciles de especificar, ya que los gerentes del negocio a menudo solo expresan las expectativas generales sobre los objetivos que el AD debe apoyar, y (iii) los sistemas de AD disponen de muchos usuarios con distintos tipos de intereses, y por tanto, con diferentes objetivos relacionados entre sí que deben ser modelados para obtener un modelo conceptual MD que las satisfaga [15].

En este contexto Giorgini, Rizzi y Garzetti [31] proponen un enfoque de ingeniería de requerimientos para AD basado en la metodología Tropos, en la que diseñan un modelo de objetivos. Otro de los enfoques propuestos es el de Salinesi y Gam [32], CADWA, que cuenta con información desde los objetivos estratégicos de la empresa, los objetivos de los analistas de información, la estructura de los sistemas transaccionales, y de los modelos de AD existentes que pueden ser reutilizables. Con estas fuentes crean un modelo de objetivos utilizando el modelo MAP. Por otro lado, Bonifati, Cattaneo, Ceri, Fuggetta, y Paraboschi [33] presentaron una metodología para el diseño del AD que nombran GQM, en la que obtienen un modelo de objetivos por medio de la refinación de los mismos.

Lamentablemente, estos enfoques no incluyen algún modelo que permita verificar el alineamiento de los objetivos con la estrategia del negocio.

\section{ENFOQUE DE INGENIERÍA \\ DE REQUERIMIENTOS PARA ALMACENES DE DATOS}

Una aproximación de ingeniería de requerimientos para AD debe facilitar la especificación de objetivos estratégicos, a partir de los cuales se obtengan

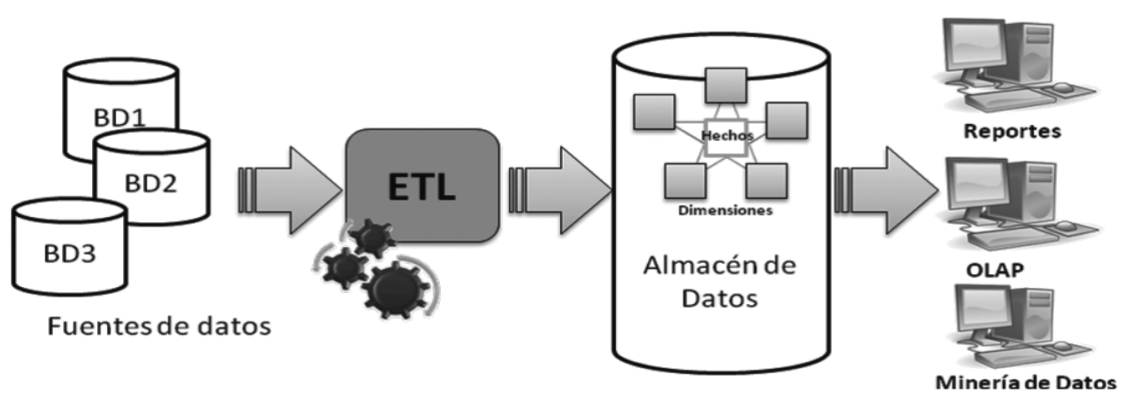

Figura 1. Proceso para extraer información desde un AD. 
aquellos requisitos de información relacionados con las medidas de interés para los procesos de negocio (contenidos en hechos) y el contexto para el análisis de estas medidas (dimensiones) que serán implementadas en dicho AD [34].

En nuestro enfoque (ver Figura 2) nos centramos en la definición de los objetivos que debe lograr cada actor (usuarios del sistema) y las relaciones necesarias para cumplir con dichos objetivos, de tal manera de conseguir un alineamiento entre estas necesidades y la estrategia organizacional. Por lo tanto, estos objetivos son los principales que la organización desea analizar mediante un AD. Los objetivos de cada actor los hemos clasificado en objetivos estratégicos, de decisión, y de información (encontramos una explicación más detallada de esta clasificación en [15]) con el fin de comprender la información que requiere el usuario para analizar los datos históricos de su trabajo.

De acuerdo con el enfoque presentado en la Figura 2, el analista deberá seguir algunas etapas para obtener los requisitos de información del AD. El proceso comienza con el análisis VMOST, que es una herramienta utilizada para obtener los elementos del plan estratégico del negocio, es decir, la misión, visión, objetivos y estrategias. En nuestra propuesta utilizamos VMOST para obtener los objetivos estratégicos, de decisión y de información que necesitan analizar los futuros usuarios del AD (en la siguiente subsección encontramos una breve explicación). Sin embargo, este análisis no asegura que los objetivos obtenidos estén alineados a la verdadera estrategia organizacional, ya que muchas veces los usuarios expresan sus propias necesidades $[9,21]$ y no las de la organización.

Para alinear cada componente obtenido a la estrategia organizacional aplicamos las reglas presentadas en BMM, ya que este es un estándar que ha sido utilizado para verificar que los elementos definidos para el plan estratégico del negocio sean los correctos (ver reglas en Figura 3). Con el fin de aplicar las reglas de BMM de manera ordenada hemos provisto un conjunto de preguntas que ayudan al diseñador a verificar el alineamiento entre los objetivos definidos para el AD y los elementos del plan estratégico de la organización. Una vez que se verifica la alineación, se continúa con la construcción del modelo de objetivos basado en $i^{*}$. El modelo $i^{*}$ contiene las necesidades de los usuarios permitiendo obtener los requisitos de información del $\mathrm{AD}$ de una manera explícita (ver detalles de su uso en [35]). Por último, cada una de las tareas y los recursos representados en el modelo $i^{*}$, deben ser incorporados en un MD.

En las siguientes secciones explicamos las etapas propuestas de la Figura 2.

\section{Análisis VMOST}

VMOST [26] es utilizado como una herramienta de análisis estratégico para una organización, ya que permite obtener diversos componentes (misión, visión, objetivos y estrategias) de la estrategia mediante un conjunto de preguntas establecidas[32] [32]. Esta técnica ha sido utilizada en $[11,36]$.

Las preguntas originales de VMOST las hemos clasificado en estratégicas, de decisión y de

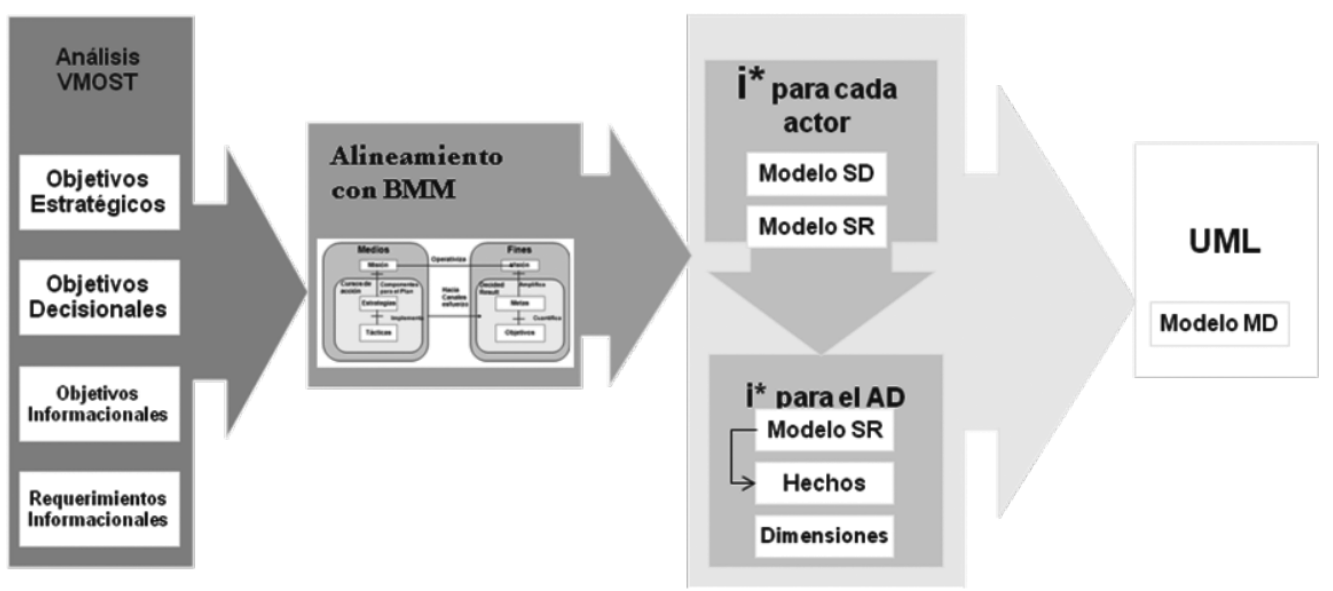

Figura 2. Propuesta de ingeniería de requerimientos para AD. 
información con el fin de guiar al diseñador para obtener los objetivos estratégicos, decisionales e informacionales del AD, que darán pie a los requerimientos de información. La Tabla 1 presenta tales preguntas.

Tabla 1. Preguntas clave para el diseñador del AD

\begin{tabular}{|l|}
\hline \multicolumn{1}{|c|}{ Preguntas Clave VMOST } \\
\hline Preguntas estratégicas \\
(1) ¿Qué objetivos debe cumplir usted para que la \\
organización compita con éxito? \\
Preguntas de decisión \\
(2) ¿Qué actividades realiza para alcanzar los objetivos \\
en la pregunta 1? \\
Preguntas de Información \\
(3) ¿Cuáles son los objetivos medibles que indican el \\
logro de los objetivos mencionados en la pregunta 1? \\
Preguntas para obtener los requerimientos de \\
Información \\
(4) ¿y qué actividades debe llevar a cabo para lograr \\
los objetivos Informacionales?
\end{tabular}

\section{Alineamiento con BMM}

El modelo BMM es la especificación del grupo de reglas de negocio BRG [27] y uno de los estándares de la OMG [37] para verificar la coherencia entre los elementos de un plan estratégico. Estos elementos facilitan la gobernanza y la orientación para el negocio.
BMM ayuda en el proceso de obtención de requerimientos para el desarrollo de sistemas, de tal manera que estos se alinean con la estrategia del negocio [38]. Además proporciona un vocabulario que es entendido por las partes interesadas para el desarrollo del negocio, y la comunicación y gestión de los planes de negocios de una manera organizada [27]. Por tanto, es una herramienta valiosa que permitirá verificar la coherencia de los objetivos planteados para el AD en el contexto estratégico de la organización. BMM ha sido utilizado en [11, 38-41].

La Figura 3 ilustra los elementos del modelo BMM y sus relaciones para apoyar el negocio.

Los "Medios" incluyen la misión, y los cursos de acción (es decir, las estrategias y tácticas). Los "Fines" incluyen la visión y los resultados deseados (es decir, metas y objetivos). En la figura también representamos los elementos de $i^{*}$ que se obtienen a partir de los componentes de BMM. En este sentido, los objetivos y estrategias de BMM servirán para identificar objetivos de $\mathrm{i}^{*}$, y las tácticas de BMM para obtener los requerimientos de información (por medio de las tareas y recursos).

BMM dispone de un conjunto de reglas pero no de una metodología que permita guiar su uso [7]. En este contexto se ha creado un conjunto de preguntas que permiten orientar al analista en el proceso de
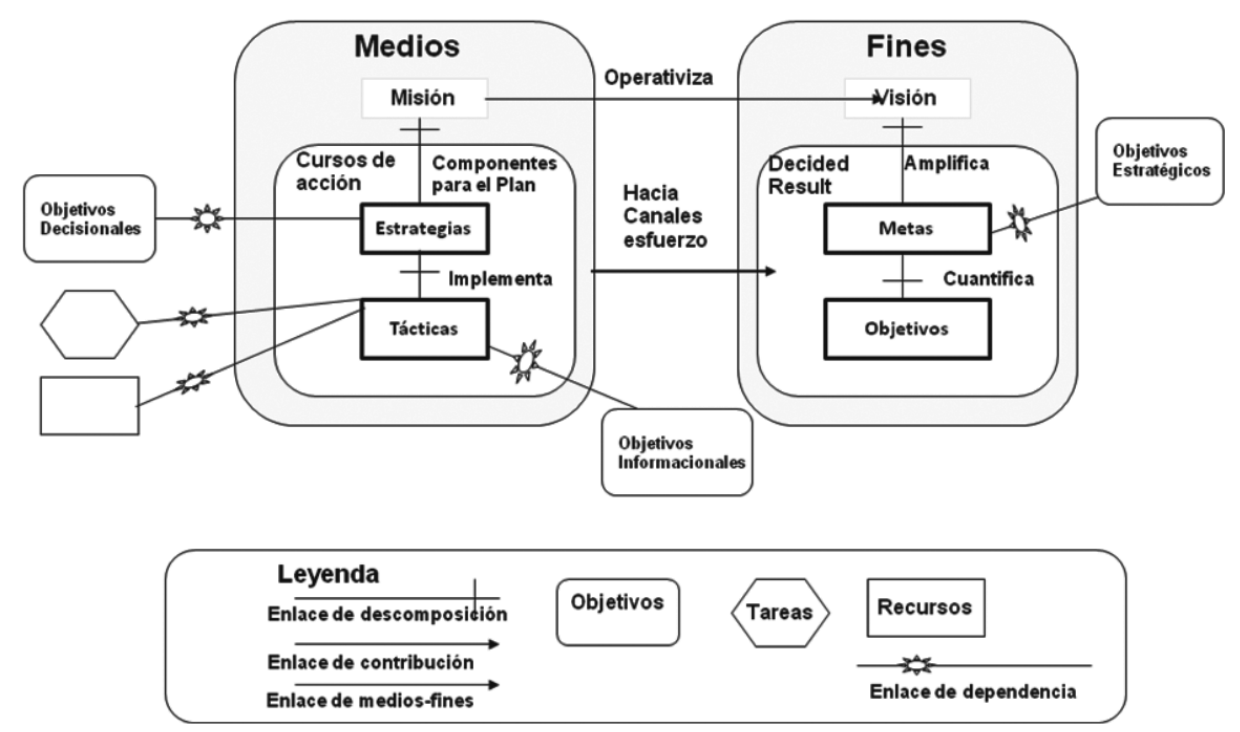

Figura 3. Modelo BMM y las relaciones con i*. 
Tabla 2. Preguntas clave para el alineamiento con BMM.

\begin{tabular}{|l|}
\hline \multicolumn{1}{|c|}{ Preguntas Clave BMM } \\
\hline (1) ¿Los objetivos estratégicos obtenidos con VMOST \\
amplifican la Visión del negocio? \\
(2) ¿Los objetivos decisionales obtenidos con VMOST \\
son componentes de la Misión del negocio? \\
(3) ¿Los objetivos decisionales permiten el logro de \\
los objetivos estratégicos? \\
(4) ¿Los objetivos informacionales implementan los \\
objetivos estratégicos?
\end{tabular}

validación de los objetivos obtenidos (desde los usuarios) con la estrategia organizacional. La Tabla 2 presenta tales preguntas.

\section{Modelo de objetivos $i^{*}$}

En [15] se diseñó un perfil de $\mathrm{i}^{*}$ para obtener los requerimientos del $\mathrm{AD}$ desde las necesidades de los tomadores de decisiones (usuarios del AD), ya que esta técnica permite representar a los actores (o usuarios) sus dependencias y la estructuración de los objetivos del negocio que pretenden alcanzar. Esta técnica consiste en dos modelos: el modelo de dependencia estratégica (SD) para describir las relaciones de dependencia entre los distintos actores en su contexto organizacional, y el modelo de motivos estratégicos (SR), que se utiliza para describir los intereses y preocupaciones de cada actor y de cómo podrían abordarse [28]. El concepto central en los modelos de $\mathrm{i} *$ son las intenciones de los actores, ya que son los agentes de la organización (es decir, los usuarios del AD), los que dependen de los objetivos a alcanzar, las tareas a realizar y de los recursos que se deben suministrar.

Para nuestro enfoque utilizamos $i^{*}$ para representar de manera gráfica los objetivos estratégicos, decisionales e informacionales de cada actor del AD y las relaciones entre los mismos. Luego se añadirá un modelo SR para el AD (considerado como un actor más del sistema) al que se le incorporan los objetivos, tareas y recursos que debe analizar. Estos recursos son obtenidos por medio de las fuentes de datos definidas en la organización.

\section{Modelado Multidimensional}

Cada una de las tareas y los recursos que se reflejan en el modelo SD para el actor AD deben ser incorporados en un modelo MD. Este modelo debe ser útil para cumplir con los objetivos del negocio. En este trabajo se utiliza el perfil UML propuesto por [15] para el diseño conceptual del AD según el paradigma multidimensional. La característica más importante de este paradigma es dividir los datos en los hechos (compuesto de las medidas) y dimensiones para proporcionar datos sobre el nivel adecuado de granularidad. Este perfil está definido por un conjunto de estereotipos y valores etiquetados de forma elegante y que representan las propiedades principales multidimensionales a nivel conceptual mediante un diagrama de clase UML (véase la Tabla 3) [42].

A continuación se describe un conjunto de directrices que ayuda a los analistas a diseñar el AD alineado a la estrategia del negocio.

Tabla 3. Principales estereotipos del perfil UML.

\begin{tabular}{|c|c|c|}
\hline Estereotipos & Descripción & Icono \\
\hline $\begin{array}{l}\text { Clase de } \\
\text { hechos }\end{array}$ & $\begin{array}{l}\text { Representa las } \\
\text { medidas del negocio }\end{array}$ & \begin{tabular}{|l|l|l|l|}
$H$ & & & \\
& & & \\
& & & \\
\end{tabular} \\
\hline $\begin{array}{l}\text { Clase de } \\
\text { dimensiones }\end{array}$ & $\begin{array}{l}\text { Representa los } \\
\text { atributos de } \\
\text { dimensión y los } \\
\text { niveles de jerarquía }\end{array}$ & \\
\hline Clase base & $\begin{array}{l}\text { Representa las } \\
\text { dimensiones de los } \\
\text { niveles de jerarquía }\end{array}$ & \\
\hline
\end{tabular}

\section{DIRECTRICES PARA EL ALINEAMIENTO}

Las siguientes directrices permiten a los desarrolladores obtener los requisitos de información del AD dentro del contexto organizacional y por tanto alineado a la estrategia del negocio. Esta guía mejora los trabajos presentados en [19-20] para el alineamiento del AD a la estrategia organizacional.

Directriz 1: Identificar los usuarios (También son nombrados Actores en el modelo $i^{*}$ ) del AD.

Directriz 2: Aplicar las preguntas de la Tabla 1 para obtener los elementos de la estrategia del negocio que necesita analizar cada usuario del AD.

Directriz 2.1: Aplicar pregunta $N^{\circ} 1$ de la Tabla 1 para obtener los Objetivos Estratégicos que debe analizar cada usuario. 
Directriz 2.2: Aplicar pregunta $\mathrm{N}^{\circ} 2$ de la Tabla 1 para obtener los Objetivos Decisionales que permiten cumplir los Objetivos Estratégicos de cada usuario. Directriz 2.3: Aplicar pregunta $\mathrm{N}^{\circ} 3$ de la Tabla 1 para obtener los Objetivos Informacionales que permiten cumplir con los Objetivos Decisionales de cada usuario.

Directriz 2.4: Aplicar pregunta $\mathrm{N}^{\circ} 4$ de la Tabla 1 a cada usuario del AD para obtener los Requerimientos de Información que permiten llevar a cabo los Objetivos de Información.

Directriz 3: Verificar el alineamiento entre los elementos estratégicos definidos por cada usuario y la estrategia organizacional. Para ello se requieren los siguientes pasos:

Directriz 3.1: Aplicar la pregunta $\mathrm{N}^{\circ} 1$ de la tabla 2 para verificar que los objetivos estratégicos amplifican la Visión del negocio.

Directriz 3.2: Aplicar las preguntas $\mathrm{N}^{\circ} 2$ y $\mathrm{N}^{\circ} 3$ de la tabla 2 para verificar que los objetivos decisionales son componentes de la Misión del negocio.

Directriz 3.3: Aplicar la pregunta $\mathrm{N}^{\circ} 4$ de la tabla 2 para verificar que los objetivos informacionales implementen los objetivos estratégicos.

Directriz 4: Crear el modelo SD de i* considerando los usuarios identificados, el actor AD y el actor fuentes de datos. Este modelo permite obtener una mirada general de lo que requiere cada usuario del $\mathrm{AD}$, y lo que requiere el $\mathrm{AD}$ de las fuentes de datos, además de mostrar las dependencias entre los mismos. El proceso de su construcción lo encontramos en [15].
Directriz 5: Crear los modelos SR de i* para cada usuario identificado. El proceso de su construcción lo encontramos en [15]. A partir de este modelo es posible determinar los requerimientos de información de los usuarios.

Directriz 6: Crear el modelo SR para el actor AD. Para ello se debe obtener los requerimientos de información, que son tareas y recursos que provienen de las fuentes de datos y que permiten analizar un objetivo de Información. Las directrices para su construcción se encuentran en [2, 19, 35].

Directriz 7: Traspasar el modelo SR del actor AD al modelo MD utilizando el perfil UML definido en [43].

La Figura 4 muestra el orden en que se deben llevar a cabo estas directrices.

Las directrices anteriores permitirán a los diseñadores del AD generar una buena posición frente a los ejecutivos del negocio para responder preguntas como:

- ¿Qué objetivos debe analizar el usuario del AD?

- ¿Cuáles estrategias de negocio se deben aplicar para cumplir con los objetivos seleccionados?

- ¿Qué objetivos no son compatibles con las estrategias?

- ¿Qué objetivos, estrategias y tácticas son afectadas si cambia algún objetivo estratégico?

- ¿Por qué tenemos este AD?

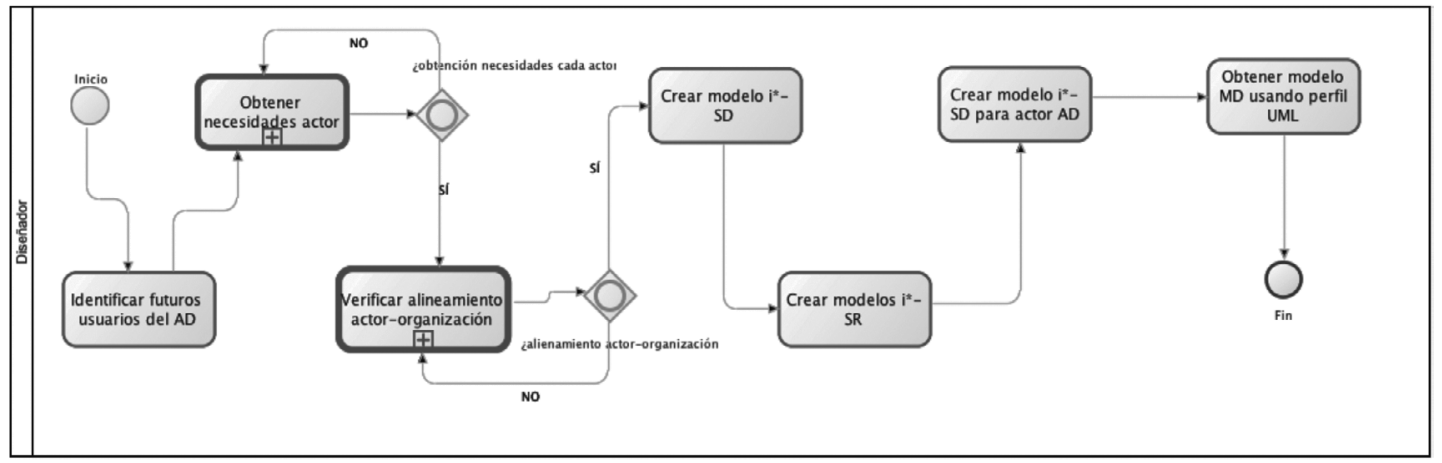

Figura 4. Proceso de ejecución de las directrices. 
- ¿Qué tareas y recursos se necesitan para desarrollar una táctica?

- ¿Cuáles son los requisitos del AD?

La trazabilidad entre los requisitos proporciona la información necesaria para responder a estas y otras cuestiones de negocios. Por tanto, este enfoque ayuda a proporcionar una manera de capturar, validar, analizar y gestionar los cambios de requisitos. Mediante el uso de esta técnica, podremos comprender mejor el quién, qué, porqué y el cómo de los requisitos del AD.

A continuación se describe un estudio de caso que utiliza las directrices formuladas para alinear los requisitos de información del $\mathrm{AD}$ con la estrategia del negocio.

\section{ESTUDIO DE CASO}

El estudio de caso de venta presentado en el libro de Kimball [44] muestra una breve descripción de una empresa minorista, que abarca tanto las ventas al por menor y el inventario. Este negocio al por menor se compone de varias tiendas de comestibles, repartidas en varias regiones. En cada tienda se venden varios productos. En el supermercado, la gestión se ocupa de la logística de los pedidos, almacenamiento, venta de productos y de maximizar las ganancias. La ganancia proviene, entre otras cosas, atrayendo a tantos clientes como sea posible en un entorno de precios altamente competitivos.

En este estudio de caso, Kimball trata con varios tipos de modelos de inventario de una tienda. Estamos interesados en el ejemplo del inventario justo a tiempo, donde se miden los niveles de inventario de todos los días y se colocan en registros separados en la base de datos. El principal objetivo de gestión es la toma de decisiones para optimizar los niveles de inventario a fin de disminuir los costos. Estas decisiones están relacionadas para asegurarse que el producto adecuado está en la tienda justo en el momento adecuado para reducir al mínimo fuera de stock y reducir así el costo de acarreo general para el inventario. Así, para una buena gestión de inventario es necesario analizar la cantidad diaria en los niveles de inventario a la mano, por producto y por almacén. El encargado del inventario también se ocupa de medir la velocidad de movimiento de inventario (cómo el producto se mueve a través de la tienda) para conocer los beneficios de las ventas. Así, el gerente necesita obtener el GMROI (Margen bruto de las existencias).

Tabla 4. Visión y Misión del negocio.

\begin{tabular}{|l|l|}
\hline Elemento & \multicolumn{1}{c|}{ Descripción } \\
\hline \multirow{7}{*}{ Visión } & $\begin{array}{l}\text { Ser la cadena de comestibles más } \\
\text { efectiva, más innovadora de la región, } \\
\text { que cuenta con los profesionales más } \\
\text { motivados y capacitados para ofrecer } \\
\text { a los clientes la mejor atención, la } \\
\text { fabricación de los mejores productos, } \\
\text { y proporciona productos diversos } \\
\text { en calidad, marcas y presentaciones } \\
\text { accesibles a todos los niveles de la } \\
\text { sociedad en el futuro. }\end{array}$ \\
\hline \multirow{5}{*}{ Misión } & $\begin{array}{l}\text { Proporcionar bienestar a nuestros } \\
\text { clientes, proveyéndoles soluciones a sus } \\
\text { necesidades y servicios de conveniencias } \\
\text { a precios accesibles, en un ambiente } \\
\text { cálido, amplio, cómodo y moderno, } \\
\text { contribuyendo de esta manera a mejorar } \\
\text { su calidad de vida. }\end{array}$ \\
\hline
\end{tabular}

Primero utilizamos la directriz $\mathrm{N}^{\mathrm{o}} 1$ para identificar los futuros usuarios (Actores) del AD. En este caso serán los gerentes de Marketing y de Inventarios.

Utilizando la directriz $\mathrm{N}^{\mathrm{o}} 2$ podemos descomponer la estrategia del negocio a partir de su Misión y Visión. Entrevistando a los Actores se obtuvieron los objetivos estratégicos, decisionales, e informacionales (ver Tabla 5). Por ejemplo, al aplicar la pregunta $\mathrm{N}^{\mathrm{o}} 1$ de la Tabla 1, ¿Qué objetivos debe cumplir usted para que la organización compita con éxito?, el gerente de Marketing respondió "aumentar el número de clientes".

Tabla 5. Objetivos de los gerentes de Marketing e Inventarios.

\begin{tabular}{|c|c|c|}
\hline Estratégicos & Decisionales & Informacionales \\
\hline $\begin{array}{l}\text { Aumentar el } \\
\text { número de } \\
\text { clientes }\end{array}$ & $\begin{array}{l}\text { Determinar } \\
\text { si la pro- } \\
\text { moción es } \\
\text { efectiva }\end{array}$ & $\begin{array}{l}\text { Analizar la } \\
\text { eficacia de las } \\
\text { promociones }\end{array}$ \\
\hline \multirow{2}{*}{$\begin{array}{l}\text { Disminuir } \\
\text { los costos de } \\
\text { inventario }\end{array}$} & \multirow{2}{*}{$\begin{array}{l}\text { Optimizar la } \\
\text { gestión de } \\
\text { inventario }\end{array}$} & $\begin{array}{l}\text { Estudiar los } \\
\text { niveles de } \\
\text { inventario }\end{array}$ \\
\hline & & $\begin{array}{l}\text { Estudiar los } \\
\text { movimientos de } \\
\text { inventario }\end{array}$ \\
\hline
\end{tabular}


Como se ha descrito en párrafos anteriores, los objetivos de la Tabla 5 deben estar alineados a la estrategia del negocio por lo que será necesario aplicar el conjunto de directrices $\mathrm{N}^{\circ} 3$.

De acuerdo con la directriz $\mathrm{N}^{\circ} 3.1$, preguntamos ¿los objetivos estratégicos obtenidos con VMOST amplifican la Visión del negocio? Con esta pregunta verificamos que los objetivos estratégicos amplifican la Visión del negocio, ya que "aumentar el número de clientes "y "disminuir los costos de inventarios" permitirán que la cadena de suministros sea más efectiva ofreciendo una mejor atención a los clientes y con precios más accesibles.

Por otro lado, al aplicar la directriz $\mathrm{N}^{\circ} 3.2$ hacemos la pregunta ¿los objetivos decisionales obtenidos con VMOST son componentes de la Misión del negocio? Con esta pregunta verificamos que los objetivos decisionales definidos son componentes de la Misión del negocio, ya que al "determinar si la promoción es efectiva" ayuda a proveer soluciones a las necesidades de los clientes, y al "optimizar la gestión de inventarios" ayuda a obtener precios más accesibles. Y con la pregunta ¿los objetivos decisionales permiten el logro de los objetivos estratégicos? Verificamos que el objetivo "determinar si la promoción es efectiva" permite al gerente de Marketing lograr de mejor manera el objetivo estratégico "aumentar el número de clientes".

Utilizando la directriz $\mathrm{N}^{\circ} 3.3$ debemos aplicar la pregunta $¿$ Los objetivos informacionales implementan los objetivos estratégicos? Así verificamos que los objetivos informacionales implementan los objetivos decisionales, ya que "analizar la eficacia de las promociones" permite "determinar si una promoción es efectiva" y "estudiar los niveles y movimientos de inventario" permite "optimizar la gestión de inventario".

De acuerdo con la directriz 4 debemos crear el modelo SD de i* con los datos obtenidos. La Figura 5 muestra un ejemplo.

De acuerdo con la directriz 5 debemos crear el modelo SR para cada actor del AD. La Figura 6 muestra el modelo SR para el gerente de Marketing.

Observamos que para determinar si las promociones son efectivas, el gerente debe analizar su

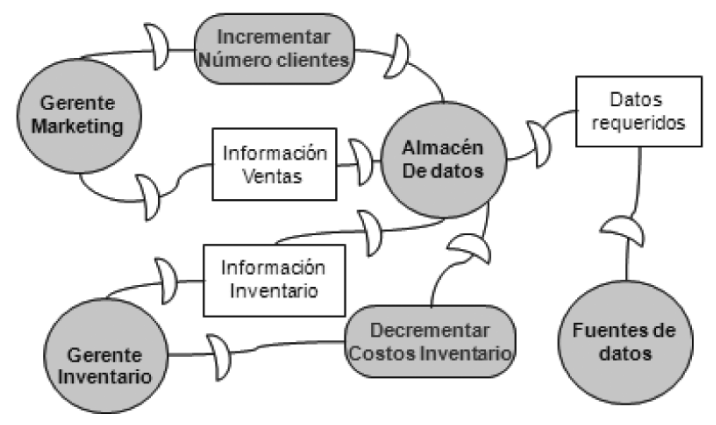

Figura 5. Modelo SD de i*.

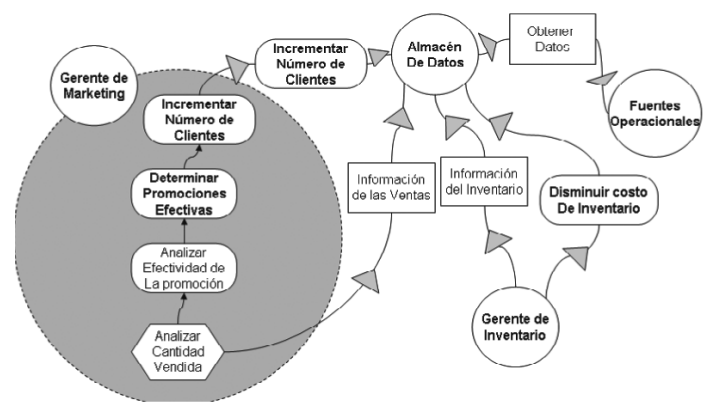

Figura 6. Modelo SR para el gerente de Marketing.

efectividad, por lo que hemos encontrado un requerimiento informacional: "Analizar efectividad de las promociones". Este requerimiento es posible implementarlo en un AD al "analizar la cantidad vendida", que es una tarea a incorporar en el modelo.

Una vez creado el modelo SR para cada actor se lleva a cabo la directriz 6, es decir, crear el modelo SR para el actor AD (el proceso para su construcción se encuentra detallado en [2, 19, 35]). Así encontramos que para "analizar la cantidad vendida", el AD debe "proporcionar información de las ventas", por lo que hemos encontrado una tabla de hechos (Ventas). Para ello, es necesario obtener la siguiente información desde las fuentes de datos: "información de productos", y la "información de promociones", que serían las dimensiones (producto, promoción). Además la cantidad vendida sería una medida. La Tabla 6 presenta un listado con los hechos, dimensiones y medidas para el AD y que son representados de manera gráfica mediante el modelo SR de i* (ver Figura 7).

Finalmente, se genera un modelo MD conceptual para el AD, tal y como muestra la Figura 8. Los detalles de la construcción de este modelo se encuentran descritos en [16, 42]. 
Tabla 6. Tablas de hechos, dimensiones y medidas.

\begin{tabular}{|c|c|}
\hline Tabla de hechos & Dimensiones (D) y medidas (M) \\
\hline \multirow{4}{*}{$\begin{array}{l}\text { Proporcionar } \\
\text { información de } \\
\text { ventas }\end{array}$} & $\begin{array}{l}\text { D: Proporcionar información } \\
\text { de productos }\end{array}$ \\
\hline & $\begin{array}{l}\text { D: Proporcionar información } \\
\text { de promociones }\end{array}$ \\
\hline & $\begin{array}{l}\text { D: Proporcionar información } \\
\text { de fechas sobre las ventas }\end{array}$ \\
\hline & M: Analizar cantidad vendida \\
\hline \multirow{7}{*}{$\begin{array}{l}\text { Proporcionar } \\
\text { información de } \\
\text { inventario }\end{array}$} & $\begin{array}{l}\text { M: Analizar los niveles diarios } \\
\text { de producto disponible en el } \\
\text { almacén (cantidad en mano) }\end{array}$ \\
\hline & $\begin{array}{l}\text { M: Obtener información del } \\
\text { último precio }\end{array}$ \\
\hline & $\begin{array}{l}\text { D: Determinar periodos de } \\
\text { tiempo }\end{array}$ \\
\hline & M: Calcular GMROI \\
\hline & $\begin{array}{l}\text { M: Calcular el total cantidad } \\
\text { vendida }\end{array}$ \\
\hline & $\begin{array}{l}\text { D: Proporcionar información } \\
\text { de tiendas }\end{array}$ \\
\hline & $\begin{array}{l}\text { D: Proporcionar información } \\
\text { de productos }\end{array}$ \\
\hline
\end{tabular}

Una vez que tenemos el esquema multidimensional, si nos remontamos al requisito de información principal antes descrito: "analizar qué cantidad de productos se venden en qué tiendas en qué días y bajo qué condiciones de promoción”, podemos ver fácilmente que este requisito puede ser respondido por el esquema de navegación multidimensional obtenido de la Figura 8. En concreto, la medida especificada en la clase de hecho Ventas, proviene de los recursos de la "cantidad vendida", y las clases de dimensión y las clases base provienen de los recursos de "producto", "fecha", "promoción", y "tienda". Para una visión general de cuáles son los elementos multidimensionales que se crean para cumplir cada objetivo, véase la Tabla 7.

\section{CONCLUSIONES}

La ingeniería de requerimientos juega un papel clave en el desarrollo de AD con el objetivo de reducir el riesgo del fracaso. Un buen método para el diseño de $\mathrm{AD}$ debe ir precedido de la obtención de requerimientos que considera las necesidades de los usuarios y la validación de dichas necesidades con la estrategia del negocio, ya que los AD son utilizados por los sistemas de apoyo a la toma de decisiones.

El trabajo provee un enfoque de ingeniería de requerimientos orientado al negocio que incluye: (1) un análisis de los componentes de la estrategia del negocio (visión, misión, objetivos

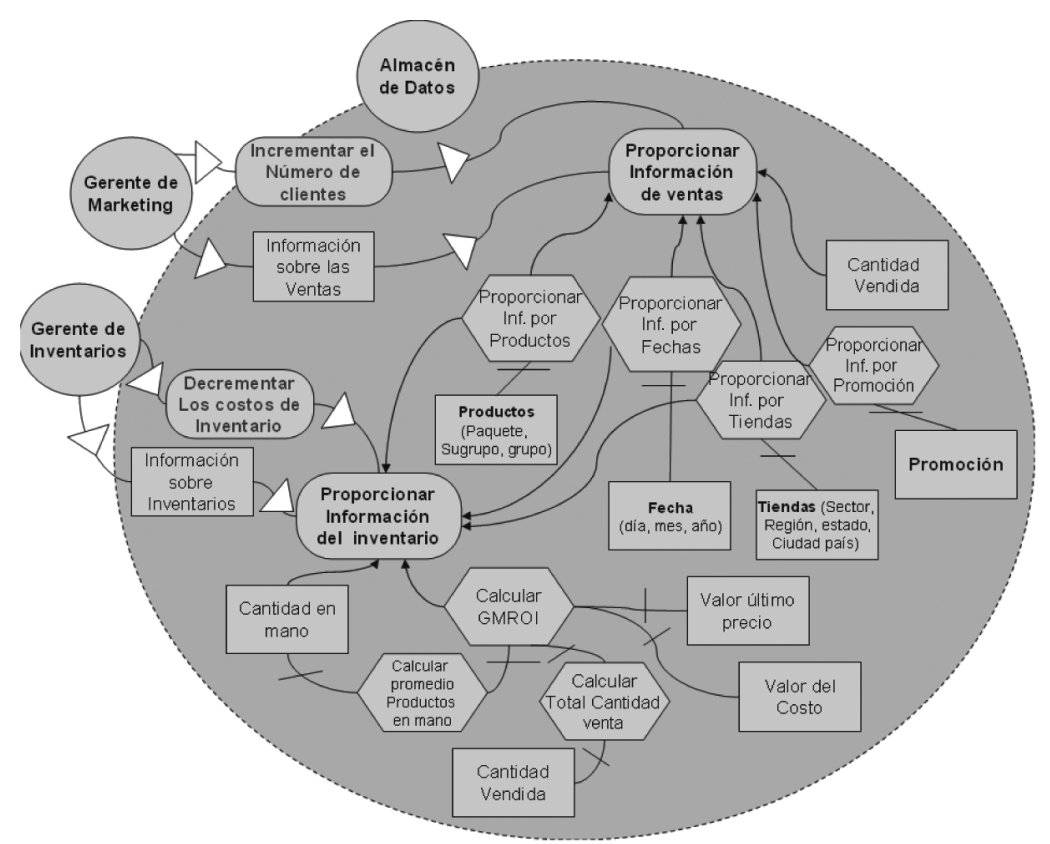

Figura 7. Modelo SR de i* para el actor AD. 


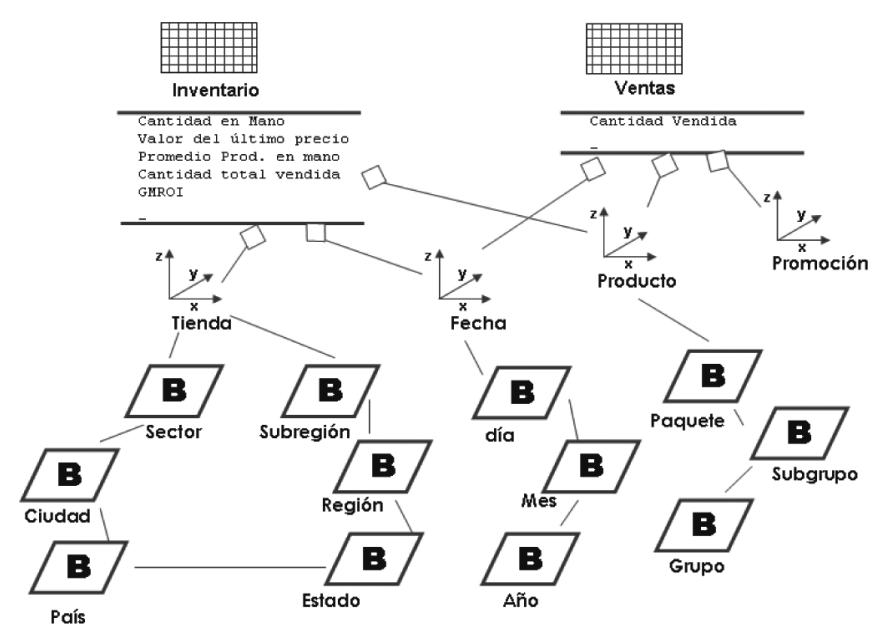

Figura 8. Diagrama MD multidimensional creado a partir del modelo de objetivos.

Tabla 7. Elementos del modelo multidimensional correspondiente a los objetivos estratégicos.

\begin{tabular}{|l|l|l|l|l|}
\hline \multicolumn{1}{|c|}{$\begin{array}{c}\text { Objetivos } \\
\text { estratégicos }\end{array}$} & $\begin{array}{c}\text { Clases de } \\
\text { hechos }\end{array}$ & \multicolumn{1}{|c|}{ Medidas } & $\begin{array}{c}\text { Clases de } \\
\text { dimensiones }\end{array}$ & $\begin{array}{c}\text { Clases Base (niveles de } \\
\text { agregación) }\end{array}$ \\
\hline $\begin{array}{l}\text { Incrementar el } \\
\text { número de clientes }\end{array}$ & Ventas & Cantidad vendida & $\begin{array}{l}\text { Tienda, fecha, } \\
\text { producto, } \\
\text { promoción }\end{array}$ & $\begin{array}{l}\text { Tienda: Código postal, } \\
\text { ciudad, país, subregión, } \\
\text { región, estado. } \\
\text { Fecha: día, mes, año. } \\
\text { Producto: paquete, } \\
\text { subgrupo, grupo. }\end{array}$ \\
\hline $\begin{array}{l}\text { Decrementar el costo } \\
\text { de inventario }\end{array}$ & Inventario & $\begin{array}{l}\text { Cantidad en mano, } \\
\text { cantidad vendida, } \\
\text { valor del último } \\
\text { precio, valor del } \\
\text { costo, cantidad } \\
\text { promedio diario en } \\
\text { mano, GMROI }\end{array}$ & $\begin{array}{l}\text { Tienda, fecha, } \\
\text { producto }\end{array}$ & $\begin{array}{l}\text { Tienda: Código postal, } \\
\text { ciudad, país, subregión, } \\
\text { región, estado. } \\
\text { Fecha: día, mes, año. } \\
\text { Producto: paquete, } \\
\text { subgrupo, grupo. }\end{array}$ \\
\hline
\end{tabular}

y estrategias) mediante un conjunto de preguntas que provee VMOST. Estas preguntas permiten a los diseñadores obtener los objetivos estratégicos, decisionales e informacionales del $\mathrm{AD}$, además de los requerimientos de información que darán pie al diseño conceptual del AD; (2) el alineamiento de los objetivos obtenidos (necesidades de los usuarios del AD) con el plan estratégico de znegocios, por medio de las reglas de BMM; (3) la representación de los objetivos obtenidos por medio del modelo i*; y (4) la representación del MD mediante un perfil UML.

Mediante el uso de VMOST y BMM, los diseñadores podrán comprender mejor el quién, qué, porqué y el cómo de los requerimientos de los usuarios, permitiendo generar una buena posición frente a los ejecutivos del negocio para responder sus preguntas. Y mediante el uso del modelado de objetivos con i* podrán comprender mejor los objetivos que desea alcanzar cada usuario y las relaciones entre los mismos.

El trabajo también incluye un conjunto de directrices que proveen a los diseñadores todos los pasos necesarios para llevar a cabo el proceso completo de diseño del AD. Como ejemplo de su uso se presenta un estudio de caso.

Como trabajo futuro se espera utilizar otros elementos de BMM en la validación de las necesidades de los usuarios. Elementos como políticas y reglas del negocio, procesos, entre otros. El desafío será representar tales elemento en el modelo $i^{*}$. 


\section{AGRADECIMIENTOS}

Este trabajo se ha realizado con el apoyo de la Dirección de Investigación, Vicerrectoría de Investigación y Postgrado de la Universidad de La Frontera, a través del Proyecto de Investigación DIUFRO DI11-0044.

\section{REFERENCIAS}

[1] O. Romero and A. Abelló. "Multidimensional Design Methods for Data Warehousing". Integrations of Data Warehousing, Data Mining and Database Technologies: Innovative Approaches, pp. 78. 2011.

[2] J.N. Mazón, J. Pardillo and J. Trujillo. "A Model Driven Goal Oriented Requirement Engineering Approach for Data Warehouses." Advances in Conceptual ModelingFoundations and Applications, pp. 255-264. 2007.

[3] C.Zambrano, M. Varas y A. Urrutia. "Enfoque MDA para el diseño de un data warehouse difuso". Ingeniare. Revista chilena de ingeniería. Vol. $20 \mathrm{~N}^{\circ}$ 3, pp. 99-113. 2012.

[4] A. Cravero and S. Sepúlveda. "A chronological study of paradigms for data warehouse design". Ingeniería e Investigación. Vol. 32 $\mathrm{N}^{\mathrm{o}}$ 2, pp. 58-62. 2012.

[5] M. B. Curtis and K. Joshi. "Developing a Data Warehouse: Some Guidelines and Suggestions". Review of Business Information Systems (RBIS). Vol. $3 \mathrm{~N}^{\circ}$ 2, pp. 13-26. 2011.

[6] V.E. Silva, J.N. Mazón, I. Garrigós, J. Trujillo and J. Mylopoulos. "Monitoring strategic goals in data warehouses with awareness requirements". Proceedings of the 27th Annual ACM Symposium on Applied Computing, pp. 1075-1082. 2012.

[7] M. Frolick and K. Winter. "Critical Factors for Data Warehouse Failure." Business Intelligence Journal http://www.tdwi.org/ research/display.aspx ?ID $=6592$. Vol. $8 \mathrm{~N}^{\circ} 1$, pp. 61-71. 2003.

[8] R. Winter and B. Strauch. "Information Requirements Engineering for Data Warehouse Systems". 2004.

[9] R. Weir, T. Peng and J. Kerridge. "Best practice for implementing a data warehouse: A review for strategic alignment". School of Computing, Napier University, 10 Colinton Road, Edinburgh EH10 5DT UK. 2003.
[10] L.-H. Thevenet. "Modeling Strategic Alignment Using INSTAL. "ER'2008 Workshops, pp. 261-271. 2008.

[11] S. Bleistein, K. Cox and J. Verner. "Validating strategic alignment of organizational IT requirements using goal modeling and problem diagrams". The Journal of Systems and Software. Vol. 79, pp. 362-378. 2006.

[12] S. Saroop and M. Kumar. "Comparison of Data Warehouse Design Approaches from User Requirement to Conceptual Model: A Survey". Communication Systems and Network Technologies (CSNT), 2011 International Conference, pp. 308-312. 2011.

[13] M. Kumar, A. Gosain and Y. Singh. "Qualityoriented requirements engineering for a data warehouse". ACM SIGSOFT Software Engineering Notes. Vol. $36 \mathrm{~N}^{\circ}$ 5, pp. 1-4. 2011.

[14] N. Prakash, D. Prakash and D. Gupta. "Decisions and Decision Requirements for Data Warehouse Systems". Information Systems Evolution, pp. 92-107. 2011.

[15] J.N. Mazón, J. Trujillo, M. Serrano and M. Piattini. "Designing Data Warehouses: From Business Requirement Analysis to Multidimensional Modeling”. REBNITA'05, pp. 44-53. 2005.

[16] J.N. Mazón, J. Trujillo and J. Lechtenbörger. "Reconciling requirement driven data warehouses with data sources via multidimensional normal forms". Data and Knowledge Engineering Vol. 63, Issue 3, pp. 725-751. 2007.

[17] J. Simonin, S. Bigaret and J. Gourmelen. "A data warehouse logical design method based on the alignment with business processes". Research Challenges in Information Science (RCIS), 2012 Sixth International Conference, pp. 1-12. 2012.

[18] R. Winter and B. Strauch. "A method for demand driven information requirements analysis in data warehousing projects". Proceedings of the 36th Annual Hawaii International Conference on System Sciences, pp. 9-14. 2003.

[19] A. Cravero, S. Sepúlveda, J.-N. Mazón, y J. Trujillo. "Alineamiento de Objetivos de la Organización: una necesidad para el Análisis de Requisitos en Almacenes de Datos”. CIBSE2010. 2010. 
[20] A. Cravero, S. Sepúlveda, J.-C. Trujillo y J.-N. Mazón. "El Alineamiento de Objetivos de la Organización como propuesta para el Análisis de Requisitos en Almacenes de Datos". Actas EIG2009. 2009.

[21] B. Cooper, H. Watson, B. Wixom and D. Goodhue. "Data Warehousing Supports Corporate Strategy at First American Corporation (FAC)". MIS Quarterly. Vol. 24. $\mathrm{N}^{\circ}$ 4, pp. 547-567. 2000.

[22] I. Singh and M. Kumar. "Evaluation of approaches for designing secure data warehouse". Proceedings of the International Conference on Advances in Computing, Communications and Informatics, pp. 69-73. 2012.

[23] G. Shanks, N. Bekmamedova and L. Willcocks. "Business Analytics: Enabling Strategic Alignment and Organisational Transformation". Proceedings of the ECIS 2012. 2012.

[24] J.N. Mazón and J. Trujillo. "An MDA approach for the development of data warehouses". JISBD'09, pp. 208. 2009.

[25] J.N. Mazón y J.C. Trujillo. "Desarrollo de modelos multidimensionales de almacenes de datos basado en MDA: del análisis de requisitos al modelo lógico". 2007.

[26] N. Rosasco and J. Dehlinger. "Business Architecture Elicitation for Enterprise Architecture: VMOST versus Conventional Strategy Capture". presented at Software Engineering Research, Management and Applications (SERA), 2011 9th International Conference. 2011.

[27] BRG. "The Business Motivation Model. Business Governance in a Volatile World". URL: www.BusinessRulesGroup.org. 2007.

[28] E. Yu. "Modelling Strategic Relationships for Process Reengineering". PhD thesis, Computer Science Department. University of Toronto, Toronto (Canada). Phd thesis. also appears as Technical Report DKBSTR-94-6. December 1994. 1995.

[29] W. Inmon. "Building the Data Warehouse." 2005.

[30] E. Kavakli. "Goal Oriented Requirements Engineering: A Unifying Framework". Requirements Engineering. Vol. $6 \mathrm{~N}^{\circ} 4$, pp. 237-251. 2002.
[31] P. Giorgini, S. Rizzi and M. Garzetti. "GRAnD: A goal-oriented approach to requirement analysis in data warehouses". Decision Support Systems. Vol. 45, pp. 4 - 21. 2008.

[32] C. Salinesi and I. Gam. "How specific should Requirements Engineering be in the context of Decision Information Systems?". Research Challenges in Information Science, 2009. RCIS 2009. Third International Conference, pp. 247-254. 2009.

[33] A. Bonifati, F. Cattaneo, S. Ceri, A. Fuggetta, and F. Paraboschi. "Designing Data Marts for Data Warehouses". ACM Transactions on Software Engineering and Methodology. Vol. $10 \mathrm{~N}^{\circ}$ 4, pp. 452-483. 2001.

[34] A. Sarkar. "Data Warehouse Requirements Analysis Framework: Business-Object Based Approach". International Journal. Vol. 3. 2012.

[35] J.N. Mazón, J. Pardillo, E. Soler, O. Glorio, and J. Trujillo. "Applying the $\mathrm{i}$ * framework to the development of data warehouses". iStar'08 3rd International i* Workshop, pp. 79-82. 2008.

[36] S. Bleistein, K. Cox, J. Verner and K. Phalp. "B-SCP: A requirements analysis framework for validating strategic alignment of organizational IT based on strategy, context, and process". Information and Software Technology. Vol. 48, pp. 846-868. 2006.

[37] OMG. "Business Motivation Model". Version 1.1 Beta Specification (convenience document with change bars). 2008.

[38] B. Berkem. "How to align IT with the canges using UML and according to BMM? Applying the "Goal Driven Development" Process on a case study using UML 2 and the BMM." Journal of Objetc Technology. Chair of Software Engineering. Vol. $5 \mathrm{~N}^{\circ} 2$, 2006.

[39] T. Roach, G. Low and J. D'Ambra. "Aligning Business Motivations in a Services Computing Design". Information Systems Development, pp. 319-330. 2011.

[40] X. Cui and R. Paige. "An Integrated Framework for System/Software Requirements Development Aligning with Business Motivations". Computer and Information Science (ICIS), 2012 IEEE/ACIS 11th International Conference, pp. 547-552. 2012. 
[41] B. Andersson, M. Bergholtz, A. Edirisuriya, T. Ilayperuma, P. Jayaweera, P. Johannesson and J. Zdravkovic. "Enterprise Sustainability through the Alignment of Goal Models and Business Models". CAiSE' 08. 2008.

[42] S. Luján-Mora, J. Trujillo and I.-Y. Song. "Multidimensional modeling with UML package diagrams". 21st Intl. Conference on Conceptual Modelling (ER2002). Vol. 2503 of LNCS. pp. 199-213. 2002.

[43] S. Luján-Mora and J. Trujillo. "A comprehensive method for data warehouse design". ProceedingsDMDW. Berlin, Germany. 2003.

[44] R. Kimball and M. Ross. "The Data Warehouse Toolkit. Second edition. John Wiley \& Sons”. 2002.
[45] N. Prakash and H. Bhardwaj. "Early Information Requirements Engineering for Target Driven Data Warehouse Development". The Practice of Enterprise Modeling, pp. 188202. 2012.

[46] V. Gupta, A. Chauhan, A. Kumar and S. Taneja. "UREM-A UML-Based Requirement Engineering Model for a Data Warehouse". Proceedings of the 5th National Conference; INDIACom-2011, Computing For Nation Development, New Delhi, India. 2011.

[47] M. Golfarelli, S. Rizzi and E. Turricchia. "Modern software engineering methodologies meet data warehouse design: 4WD". Data Warehousing and Knowledge Discovery, pp. 66-79. 2011. 